\title{
Evaluating Fatigue, Stress, Workload and Situation Awareness Among Electrical Substation Construction Workers
}

\author{
Ahmed Ali Bayounis (Corresponding author) \\ Department of Industrial Engineering, Faculty of Engineering \\ King Abdulaziz University \\ PO Box 80200, Jeddah 21589, KSA \\ Tel: +966 - 555700770 E-mail: a.a.bayounis@gmail.com \\ Dr. Abdulrahman Basahel \\ Department of Industrial Engineering, Faculty of Engineering \\ King Abdulaziz University \\ PO Box 80200, Jeddah 21589, KSA
}

Tel: +966 (12) 6400000 Ext. 72398 E-mail: ambasahel@kau.edu.sa

\author{
Received: April 7, 2020 \\ Accepted: May 5, 2020 \\ Published: May 14, 2020 \\ doi:10.5296/jss.v6i1.16808 \\ URL: https://doi.org/10.5296/jss.v6i1.16808
}

\begin{abstract}
Many accidents occur due to various factors when undertaking construction projects. One of the most important achievements when undertaking a construction project is to accomplish the project without major accidents. This paper studies the effect of stress, fatigue and workload on construction contract worker in term of their safety performance results using the Situation Awareness Rating Technique (SART), with reference to how it relates to the root causes of accidents. Investigating the factors associated with accidents helps to identify and minimize the associated mistakes or causes and reduce or avoid accidents and improve safety performance in construction projects. Data were collected using a SART survey of employee of 18 contractors working on construction of electrical substations in the western area of Saudi Arabia. It was found that most accidents occurred at the worker level because of their lack of awareness and training. It is apparent that SART affects the safety performance of
\end{abstract}


contractors and it varies with the position, age and experience of workers. To implement a safe working environment free of accidents, it is necessary to improve all job levels according to their insufficient area and activate a full safety management system with periodic reviews to improve it and make it more effective.

Keywords: Safety, Construction Safety, Situation Awareness, Fatigue, Stress, Workload

\section{Introduction}

Health and safety is relevant to all types of industries but especially in the construction industry. Safety has been a primary matter considered by risky sectors when it comes to accidents. Although, significant improvements have been made in term of health and safety performance levels in developing countries, the construction industry continues to lag behind other industries (Alhajeri, 2011). In fact, construction projects have injury and fatality records that make construction one of the riskiest industries. Based on accident records, weak technical designs (e.g., poor compliance with regulations and different workers' behaviors and attitudes toward safety) have been addressed by applying new techniques. Nevertheless, it is apparent that many accidents continue to occur and their causes are rarely the result of technical failures. However, preventing accidents by implementing strong safety management systems throughout organizations should be a main concern for stakeholders (Cheng, Ryan, \& Kelly, 2012). Comparing two similar organizations (one that had implemented a safety management system and one that had not), it was found that the one that had implemented the system recorded much better performance indicators (Bottani, Monica, \& Vignali, 2009).

The impairment of safety in construction projects is a problem that affects all stakeholders. Given the wide variety of automated construction tools around the world (especially in the Kingdom of Saudi Arabia, which uses labor-intensive construction methods), a high rate of accidents and fatalities occur annually. However, worldwide problems regarding safety in construction occur at similar rates across countries; as such, impaired safety in construction projects appears to be a universal problem. As a result of the growth in construction development, high numbers of injuries have been reported in the construction sector (GOSI, 2013-2019). In 2013, the number of injuries in the Saudi construction industry totaled 26,700, which equates to nearly $51 \%$ of all occupational injuries among all industries (GOSI, 2013-2019). This percentage remained high and then fell to approximately $45 \%$ in 2016 and 2017 (GOSI, 2013-2019). In 2018, Saudi Arabia has implemented construction safety development in response to strict controls imposed by various government agencies. Since then, the number of injuries in the construction industry has stood at 11,010, which equates to less than $40 \%$ of all occupational injuries across all industries (GOSI, 2013-2019).

Construction projects have a high-risk potential and fatality rate relative to other industries in the Kingdom of Saudi Arabia. Accidents are economically very costly to society in terms of cost and lost time and productivity. Additionally, associated social costs include distress, pain and suffering for the affected families as well as wider society. Notable changes have been made to reduce the number of accidents over the years, but a significant number of accidents could nevertheless be avoided if suitable procedures were implemented, which require considerable effort. 
Electrical substations are among the biggest and most expensive construction projects in Saudi Arabia. Accordingly, in 2012, these projects implemented a new health and safety management system. After several years of applying the system, eight major accidents occurred involving construction projects during 2017 (a major accident is regarded as one that causes serious injury of fatality), most of which involved falling from a height. Previous research has indicated that most accidents in construction projects are due to falls (Huang \& Hinze, 2003). Other common accidents involve being hit by falling or moving objects, electrical shocks and burns. Therefore, construction projects involving electrical substations have been scoped for potential hazards that should be avoided.

One of the strategic objectives of this study is to suggest ways to enhance safety levels in work environments to achieve a high standard of safety. The main goal of this study is to evaluate the safety performance level over the last five years. A questionnaire was developed to define the factors that affect accident rates in construction projects. In addition, data were analyzed to define the most common hazards. Moreover, root cause analysis for serious accidents in construction projects was developed to determine the correlation with the safety system.

In this paper, various factors that influence accidents and near misses will be studied to improve the construction safety management system (Basso et al., 2004). Fatigue, stress and workload are among the main factors that affect safety implementation through various subfactors such as commitment, policy, regulation, etc. (Basahel \& Tajuddin, 2019). Workplaces are another factor affected by worker actions, behaviors or skills. The workplace factor is defined as the area and environment of the worksite. Equipment and material factors are concerned with the tools and equipment used on the worksite (Haslam, Hide, Gibb, \& Gyi, 2005).

Studies have shown that performance is affected by physical and mental demands, which can lead to accidents or failures (Basahel \& Tajuddin, 2019). Physical and mental demands related to stress and fatigue associated with excessive workloads reflect on workers' behavior (OSHS, 2003). McLauglin (2007) developed a model explaining the impact that changes in fatigue, stress and workload have on performance, revealing a significant relationship. However, low situation awareness results in more accidents by increasing unsafe acts or work conditions as a result of stress (Sneddon, Mearns, \& Flin, 2012).

The aim of this paper is to study the effect of mental and physical demands of stress and fatigue on workers' safety and performance. The paper focuses on elements that affect safety performance or culture. Workers' cultures are determined by observing their reactions to certain situations with regards to safety (Almalki et al., 2019). The lives of workers are the most important resource in any organization, and construction projects present a high degree of potential risk. More specifically, this work highlights the factors affecting workers' performance in electrical substation construction projects in the western region of Saudi Arabia using a situation awareness technique.

\section{Methodology}

This section defines and explains the methods and tools used to collect and analyze the data 
to achieve the research aims and objectives. A detailed explanation of the survey used and how to determine its results is provided. In addition, details of the method used to collect the data and determine the sample size are provided.

Statistical analysis is used to categorize the different types of accidents and to classify accident factors. A survey was developed and distributed to participants at the managerial and employee level. The survey sought information assessing the occupational health and safety system and worker performance.

The situation awareness survey was conducted for workers across various projects and contractors. Situation awareness tools measure the level of awareness among workers, supervisors and managers regarding mental demands, physical loads, stress and fatigue.

\subsection{Data Collection}

Data for all major accidents from 2014 until 2019 at all electrical substation construction projects in the western area were collected. It was found that 20 major accidents resulted in serious injury or death.

Detailed data for 2018 and 2019 were prepared to determine the number of major and minor injuries, observations or near misses and to audit the results and compare them overall as well as separately for the big contractors.

In addition, a survey was conducted to evaluate the mental and physical demands related to stress, fatigue and degree of situation awareness for each contractor. Situation awareness is essentially what psychologists call perception or attention. Situation awareness is a person's response to problems or accidents that occur suddenly. Hence, continuously monitoring situation awareness entails determining what is occurring in the work environment to infer what might happen in the future and how it is affected by stress, fatigue and workload.

\subsubsection{Questionnaire}

A questionnaire was used to collect data to measure the impact of fatigue, stress and workload on situation awareness and the root causes of accidents. To determine the sample size for the study, observations were held for 30 days to calculate the total population. The sample size was determined as 136 , which represents $10 \%$ of the total population, when applying a 95\% confidence level and 10\% margin of error. The survey was completed by 136 personnel holding different positions within the company. The questionnaire comprised six main parts and the reliability of the survey according to Cronbach's alpha was $80.4 \%$.

The general information contains the details used for classification, such as the contractor's name, job, age, and level of experience. Because the participants were ensured confidentiality when completing the survey, providing their names was optional.

SART is a multidimensional measurement that contains a number of questions that have multiple-choice responses. Ten measurements are used in the current approach, whereby participants appraise each measurement based on a scale from 1 to 7 (Salmon et al., 2009). For example, to measure the complexity of a situation, the question was "How complicated is the situation?" Participants ranked the complexity from 1 (low) to 7 (high). The SART 
measurements can be consolidated into the faster form of the SART. The SART uses ten measurements: instability of the situation, complexity of the situation, variability of the situation, arousal, concentration of attention, division of attention, spare mental capacity, information quantity, information quality, and familiarity with the situation (Gheisari, Irizary, \& Horn, 2010).

Mental demand (NASA-TLX scale) is a multidimensional rating technique that gives a calculated final score based on the median of six subcriteria, in which each subcriterion has its own weight and specifications to measure the workload (DiDomenico \& Nussbaum, 2008). These subcriteria are mental demands, physical demands, temporal demands, performance, effort and frustration level. The scale ranged from 0 (low overall workload) to 10 (high overall workload).

Physical demand (Borg-CR10 scale) is a general strategy for estimating conceptions and experience thorough pain and diligence (DiDomenico \& Nussbaum, 2008). The scale is used for many reasons, but it is primarily used for estimating physical pain on a scale from 0 (low overall workload) to 10 (high overall workload).

Stress at work is defined as workers being unable to perform their duties in the work environment. This section of the survey determines how people react to their work environment (OSHS, 2003). Each item (e.g., "Too many meetings") has a scale from 0 (strongly disagree) to 5 (strongly agree).

Fatigue at work results in a failure to perform usual practices or react to specific instructions due to over exertion. This section of the survey can determine the ability of workers to respond to a work situation. Participants ranked each statement (e.g., "I can concentrate well") according to their feelings whilst working (OSHS, 2003) on a scale from 0 (strongly disagree) to 7 (strongly agree).

\section{Result \& Discussion}

This section discusses the data and the relationships between the data and the results of the questionnaire to arrive at valuable outcomes.

\subsection{Questionnaire Result and Analysis}

This section discusses the main outcomes of the questionnaire after analysis and the relation of the questionnaire to the data.

\subsubsection{Comparison of Main Outcomes}

It was noticed that the effect of job category influenced the survey results. Managerial level respondents had the highest SART scores for stress and mental demands, whereas workers had the lowest. On the other hand, worker level had the highest results for fatigue and physical demands (from NASA-TLX), whereas the managerial level had the lowest. Meanwhile, the supervisor level was consistently in between the two other levels. In addition, it was noticed that for most results between stress and fatigue, as stress increased, fatigue also increased. This is because stress affects both the brain and body and makes people work harder, thereby increasing fatigue. In addition, the survey results provide insight into the 


\section{Macrothink}

Journal of Safety Studies

ISSN 2377-3219

2020, Vol. 6, No. 1

relationship between SART and each part of the survey. First, it is apparent that the SART level depends on the job category; the managerial level has the highest SART, whereas workers have the lowest and supervisors are in between. In addition, a positive relationship exists between SART and both age and experience because additional age or experience increases the SART level. However, the correlations between situation awareness and stress, fatigue and NASA-TLX (workload) were significant $(\mathrm{p}<0.05)$.

To test the impact of workload on situation awareness, stress and fatigue, an ANOVA test was conducted. It was found that mental demand had a significant impact on both situation awareness and stress, with a significance value less than 0.05 , while it had no significant impact on fatigue. Furthermore, it was found that physical demand had a significant impact on both situation awareness and fatigue, with a significance value less than 0.05 , while it had no significant impact on stress.

\subsubsection{Impact of Stress, Fatigue and Workload on Situation Awareness}

The impact of workload (mental and physical demands), stress and fatigue have been determined. For the ANOVA test with a significance value less than 0.05, SPSS analysis confirmed fatigue and mental and physical demands have a significant impact on situation awareness. Furthermore, the impact of stress on situation awareness was not significant (the value was 0.24 , which is greater than 0.05 ). 


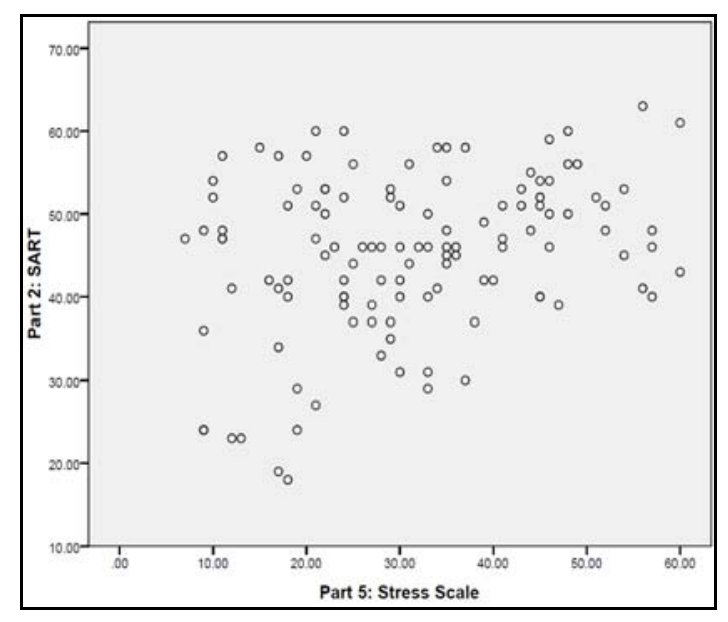

Figure 1. Relation between situation awareness and stress

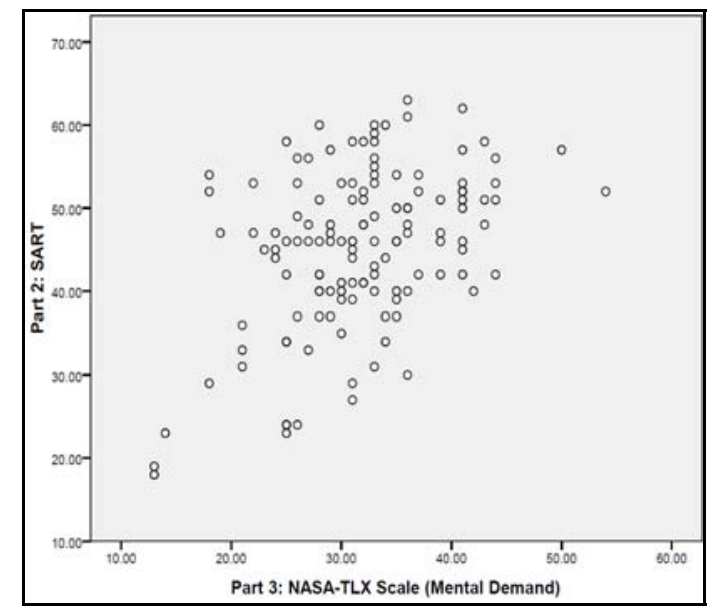

Figure 2. Relation between situation awareness and workload

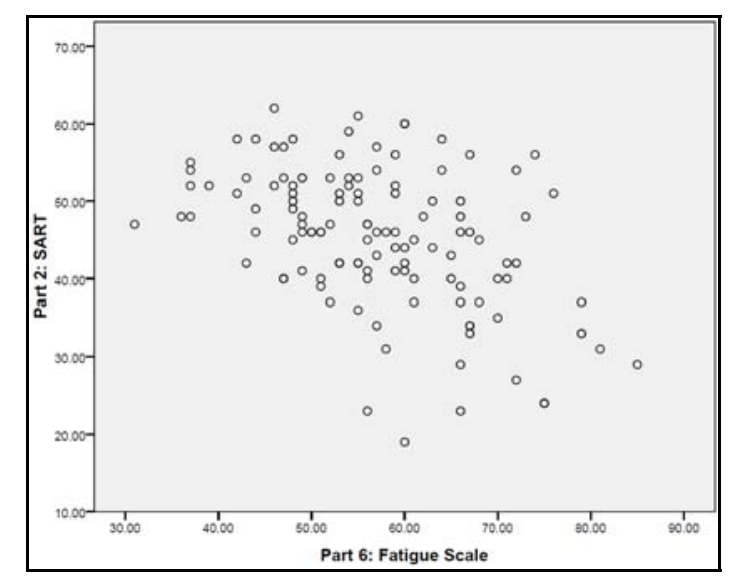

Figure 3. Relation between situation awareness and fatigue

Figures 1 and 2 illustrate the positive relationship stress and workload (represented as mental demand) have with situation awareness. Meanwhile, Figure 3 presents the negative relationship between fatigue and situation awareness.

\subsection{Impact of SART on Performance}

To make comparisons between contractors, we need to evaluate each one's performance and match it with the survey results. Two methods were used to evaluate contractor performance: First, we used an audit with detailed criteria that had been done on an annual basis and concentrates on the management system and involvement and working procedures. The other method involved greater consideration of the actual working environment and practices and is based on the contractor's monthly evaluation using summary criteria of the annual audit criteria. 


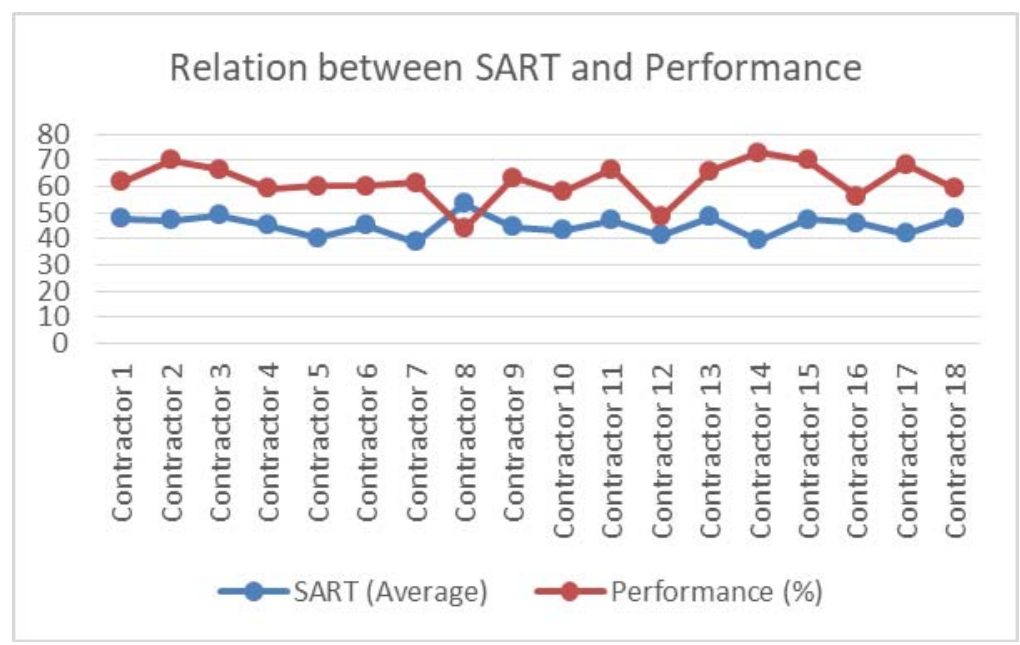

Figure 4. Relationship between SART and contractors' performance

Figure 4 shows a relationship exists between SART and contractors' performance, but some contractors have a few anomalous values, which if removed from the graph would result in a positive relationship between performance and SART. The results show some contractors have high performance scores but low SART results. On the other hand, some contractors have low performance scores but high SART results. Both cases show a mistake exists in the safety management system. High performance and low SART occurred because the contractors were willing to improve and trying to implement the safety system, whereas the workers have low SART results, which affects the overall SART result. Low performance and high SART occurred because the contractor has a good awareness level but is careless when applying the safety requirements. Finally, it is apparent that situation awareness affects the performance of contractors. Stress, fatigue and workload all have an impact on situation awareness; therefore, stress, fatigue and workload affect the performance of workers.

Based on the survey results, certain factors can increase the likelihood of an individual being exposed to accidents. Therefore, in our opinion, based on the results of this study, those factors must be taken into consideration by management. It is believed that in spite of a lack of data at hand posed by the situation awareness survey of the very group that experienced accidents, we can correlate that these results or outcomes are similar if not exactly the same factors that affect the situation prior to an accident.

\section{Conclusion}

This paper addressed the evaluation of management safety systems by analyzing accident factors and finding the root causes of accidents. In addition, it determined the level of situation awareness at each level in the working environment.

Many studies have shown that to improve health and safety, employees must change their behaviours and become more motivated. It is apparent that these efforts alone are not sufficient to eliminate safety issues in construction projects. Moreover, some studies have investigated health and safety in construction projects in developed countries. In most of these studies, researchers either set up a new procedure or improved an existing system. 
Between 2014 and 2019, the records showed that no progress was made in reducing the number of accidents even after implementing the 5-Star management system. Meanwhile, from accident investigation reports it was determined that the recommendations of investigators had become more specific and professional over time. This development suggests that the implementation had improved over time and corrective action had been taken continuously to cover gaps that emerged in terms of implementation. On the other hand, even after implementing the safety system, a significant number of accidents continued to occur.

The impact of workload and fatigue on situation awareness was direct. Meanwhile, an indirect impact as a result of stress was observed. The analysis of the situation-awareness questionnaire results showed that the relationship between different job categories in most of the survey was significant except in terms of physical demands. However, a positive relationship was observed between situation awareness and mental demand, but no significant relationship was seen between situation awareness and physical demand. In addition, it showed that as the situation awareness level increased, work stress also increased, while fatigue decreased. This result explains why management has a high level of situation awareness and high stress but a low level of effort and fatigue, whereas workers have low situation awareness levels and low stress but high levels of fatigue.

Almost all major accidents occurred at the worker level due to a variety of factors, most of which relate to a lack of awareness of their rights and safety and their training to perform tasks safely according to safe work procedures. However, mental demands, stress and fatigue are related to the SART level. Furthermore, the SART level affects safety performance unless the safety system has not been implemented sufficiently.

Accidents are one of the most disturbing things in any project. In addition, injuries make completing a project more difficult, while major injuries or fatalities make it considerably more complex. To avoid accidents and derive more benefit from implementing the safety management system, the system should be comprehensive and integrated with the management system. Accidents usually happen due to insufficient instruction, implementation procedures, penalties, reviews or training. It is necessary to address all of the gaps identified to make the safety management system more effective. A concerted awareness campaign should be undertaken across all work levels in addition to training in the system and its procedures with supervision and guidance. Furthermore, applying penalties can compel people to take their training and responsibilities seriously, follow instructions and implement the system properly.

The limitation in the current study is that the sample size was not very large, and the type of construction project considered in this study is an electrical substation. Therefore, the results of the current study cannot necessarily be generalized to all other types of construction projects such as manufacturing and healthcare. Additionally, the methods used in this study mainly focused on subjective techniques such as TLX-NASA, SART and fatigue and stress surveys, in addition to the number of accidents. Objective measures such as heart rate, energy expenditure, breathing rate and number of lost workdays could add great value to determining the extent of the impact of fatigue, stress and workload on situation awareness and 
performance.

Conflicts of interest: The authors declare no conflict of interest.

Acknowledgement: The authors would like to express their gratitude to all of the projects supervisors and workers who participated in the study.

Financial Support: The research is not financed by any authority.

\section{References}

Alhajeri, M. (2011). Health and safety in the construction industry: Challenges and solutions in the UAE. Coventry University.

Almalki, B., Zakeria A., Balkhoyour M., \& Ahmad I. (2019). Safety Climate and Culture Review: A Case Study of Water and Power Project. Journal of Safety Studies, 5(1).

Basahel, A., \& Tajuddin, F. (2019). Ergonomics Factors, Mental and Physical Loads Among Health-Care Workers.

Basso, B., Carpegna, C., Dibitonto, C., Gaido, G., Robotto, A., \& Zonato, C. (2004). Reviewing the safety management system by incident investigation and performance indicators. Journal of Loss Prevention in the Process Industries, 17(3), 225-231. https://doi.org/10.1016/j.jlp.2004.02.004

Bottani, E., Monica, L., \& Vignali, G. (2009). Safety management systems: Performance differences between adopters and non-adopters. Safety Science, 47(2), 155-162. https://doi.org/10.1016/j.ssci.2008.05.001

Cheng, E., Ryan, N., \& Kelly S. (2012). Exploring the perceived influence of safety management practices on project performance in the construction industry. Safety Science, 50(2), 363-369. https://doi.org/10.1016/j.ssci.2011.09.016

DiDomenico, A., \& Nussbaum, M. (2008). Interactive effects of physical and mental workload on subjective workload assessment. International Journal of Industrial Ergonomics, 38, 11-12. https://doi.org/10.1016/j.ergon.2008.01.012

Gheisari, M., Irizary, J., \& Horn, D. B. (2010), Situation Awareness Approach to Construction Safety Management Improvement. Conference: Proceedings of 26th Annual ARCOM Conference. CONECTech Lab, School of Building Construction, Georgia Institute of Technology, USA.

GOSI (2013-2019). Annual Report. Retrieved March 1, 2020, from https://www.gosi.gov.sa/GOSIOnline/Open_Data_Library?locale=ar_SA

Haslam, R., Hide, S., Gibb, A., \& Gyi, D. (2005). Contributing factors in construction accidents. Applied $\quad$ Ergonomics, 36(4), 401-415. https://doi.org/10.1016/j.apergo.2004.12.002

Huang, X., \& Hinze, J. (2003). Analysis of Construction Worker Fall Accidents. Journal of Construction Engineering and Management, 129(3). 


\section{Macrothink}

Journal of Safety Studies

ISSN 2377-3219 2020, Vol. 6, No. 1

McLauglin, J. L. (2007). Stress, Fatigue Andworkload: Determining the Combined Affect on Human Performance. University of Central Florida.

Occupational Safety and Health Service. (2003). Healthy Work Managing stress and fatigue in the workplace (1st ed.). Department of Labour. Wellington, New Zealand.

Salmon, P., Stanton, N., Walker, G., Jenkins, D., Ladva, D., Rafferty, L., \& Young, M. (2009), Measuring Situation Awareness in complex systems. Comparison of measures study, 39(3). https://doi.org/10.1016/j.ergon.2008.10.010

Sneddon, A., Mearns , K., \& Flin, R. (2012). Stress, fatigue, situation awareness and safety in offshore drilling crews. Safety Science, 1-9. https://doi.org/10.1016/j.ssci.2012.05.027

\section{Copyright Disclaimer}

Copyright for this article is retained by the author(s), with first publication rights granted to the journal.

This is an open-access article distributed under the terms and conditions of the Creative Commons Attribution license (http://creativecommons.org/licenses/by/4.0/) 